\title{
Practice guidelines for liver biopsy
}

\author{
A Buckley MD FRCPC, D Petrunia MD MSc FRCPC
}

$\mathrm{P}$ ractitioners have seen a number of advancements in the assessment of hepatic disease. Significant improvements in serological testing and genetic screening, and numerous advances in diagnostic imaging, including magnetic resonance imaging and cholangiopancreatography, have improved diagnostic capabilities. These improvements notwithstanding, histological assessment of the liver via biopsy remains important in the diagnosis and follow-up of acute and chronic hepatic disease.

The procedure of liver biopsy has not changed greatly in the past 50 years. The physician can choose from assessment with fine needle aspiration techniques with fewer attendant complications but with the limitation of cytology assessment only. Histological assessment with a variety of manually inserted or spring-loaded, gun-driven needles is more often required. Liver biopsies may be obtained blind, ie, by clinical estimation of organ location; by using radiological guidance with computerized tomography or ultrasound; via the transvenous (transjugular/transfemoral) route in patients with contraindications to percutaneous biopsy; or via direct vision, surgically or laparoscopically in certain situations.

\section{INDICATIONS}

Because needle core biopsy of the liver is associated with a small but definite risk, biopsy should only be performed when the findings assist in the management of the patient. With this stated, the following are generally accepted indications for percutaneous liver biopsy (1).

- Elevated liver enzyme levels with no explanation (ie, rising enzyme profile or initial marked enzyme elevation)

- Elevated aminotransferase levels for six months or longer

- Hepatomegaly of unclear etiology

- Assessment of response to therapy of chronic liver disease

- Unexplained jaundice without evidence of extrahepatic obstruction

\section{SPONSORS AND VALIDATION}

This practice guideline was developed by Dr A Buckley and Dr D Petrunia and was reviewed by

- Practice Affairs Committee - Dr A Cockeram (Chair), Dr A Buckley, Dr T Devlin, Dr J McHattie, Dr E Semlacher, Dr A Tavenor and Dr M Tourigny.

- Canadian Association of Gastroenterology (CAG) Governing Board

- Canadian Association for Study of the Liver Dr G Levy and Dr HWH Sullivan

- Monitoring of hepatotoxicity of drug therapy

- Fever of unknown origin

- Liver transplant evaluation

The prebiopsy work-up of a patient requiring liver biopsy should include informed consent, including an explanation of the risks of the procedure and the following laboratory tests obtained within one week before biopsy.

- Prothrombin time-international normalized ratio and partial thromboplastin time.

- Platelet count

- Hemoglobin

- Blood urea nitrogen and creatinine

- Group and screen

- Bleeding time if acetylsalicylic acid (ASA)/nonsteroidal anti-inflammatory drug (NSAID) status is uncertain

Patients should be advised to avoid ASA and NSAIDs for one week before biopsy. Anticoagulants should be stopped for any liver biopsy.

The route of biopsy should be governed by the clinical situation. Percutaneous liver biopsy by experienced personnel is most often done. Use of ultrasound or computerized tomography to guide biopsies, although frequently used, has 
not been conclusively proven to increase diagnostic yield or to reduce complications significantly. Transvenous biopsy by skilled physicians is available for patients in whom coagulopathy or ascites preclude a traditional percutaneous approach. Laparoscopically or surgically guided biopsies may be obtained if laparoscopy and surgery are being done for other reasons or if percutaneous biopsy is inconclusive.

Postbiopsy management should include a recording by the physician performing the biopsy of how many 'passes' were made, any medications administered and any apparent complications. Suggested postbiopsy orders are that the patient have complete bed rest for a minimum of $2 \mathrm{~h}$, and that vital signs, including blood pressure, pulse and respiration, be obtained every 15 mins for $1 \mathrm{~h}$ and then at regular intervals until discharge.

Same day discharge is the norm in many centres, although it should be recognized there is a small admission rate to hospital for complications. Patients should be discharged with follow-up instructions and should be instructed to avoid NSAIDs, ASA, warfarin, ticlid and any other anticoagulants for one week.

\section{CONTRAINDICATIONS}

Contraindications to percutaneous liver biopsy include (1)

- an uncooperative patient;

- impaired coagulation;

- severe uncorrected anemia;

- significant ascites;

- infection in the path of the needle;
- suspected extrahepatic biliary obstruction of high grade;

- cholangitis;

- echinococcal cysts;

- no safe unobstructed access route to biopsy;

- leukemia and myelofibrosis; and

- uremia.

\section{COMPLICATIONS}

Complications may be divided into minor complications and major complications. Minor complications include pain and transient hypotension (3.5\% to $33 \%$ if pain included). Major complications include significant hypotension, pneumothorax, hemothorax, hemoperitoneum, hemobilia, significant intrahepatic hematoma, cardiac arrhythmia, gallbladder perforation and biopsy of other organs such as kidney or pancreas (2).

The most significant complication of liver biopsy is death, with reports ranging from $0 \%$ to $0.33 \%$ in the literature.

\section{CONCLUSION}

Liver biopsy remains an essential investigation for the diagnosis of liver disease. With strict attention to indications and contraindications, liver biopsy can be regarded as a safe and useful procedure.

\section{REFERENCES}

1. Tobkes AI, Nord HJ. Liver biopsy: Review of methodology and complications. Dig Dis 1995;13:267-74.

2. Stotland BR, Lichtenstein GR. Liver biopsy complications in routine ultrasound. Am J Gastroenterol 1996;91:1295-6.

\section{CAG PRACTICE GUIDELINE DISCLAIMER}

This clinical practice guideline has been developed by the author on behalf of the Canadian Association of Gastroenterology $(\mathrm{CAG})$ in order to outline the clinical approach to management problems or training issues. After preparation by the author, based on a review of the literature, each guideline is reviewed extensively by the CAG Practice Affairs Committee, composed of practitioners from across Canada. Changes are made, and once the guideline is felt to be appropriate, it is then circulated for further review by recognized Canadian experts and then amended further. Finally, the guideline is presented to the CAG Governing Board for further review and final approval. Practice guidelines are intended to give an understanding of a clinical problem and outline one or more preferred approaches to investigation and management of the problem. While practice guidelines are intended to be useful to all physicians, it is recognized that specialists may rely less on practice guidelines than those in more general practice. These guidelines are intended to give a practical approach to a problem based on the current literature but are not intended to be state-of-the-art reviews with extensive references.

Practice guidelines are developed to be of assistance to practising clinicians and are not intended to be the only approach to the management of clinical problems, nor are they intended to be considered a standard of care. The CAG Practice Affairs Committee recognizes that clinical circumstances may at times justify an approach different from that outlined in a practice guideline. It is also recognized that new developments in medical research and clinical practice may require subsequent changes to the practice guideline. 


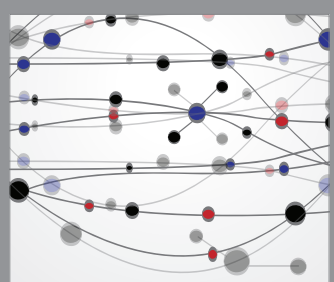

The Scientific World Journal
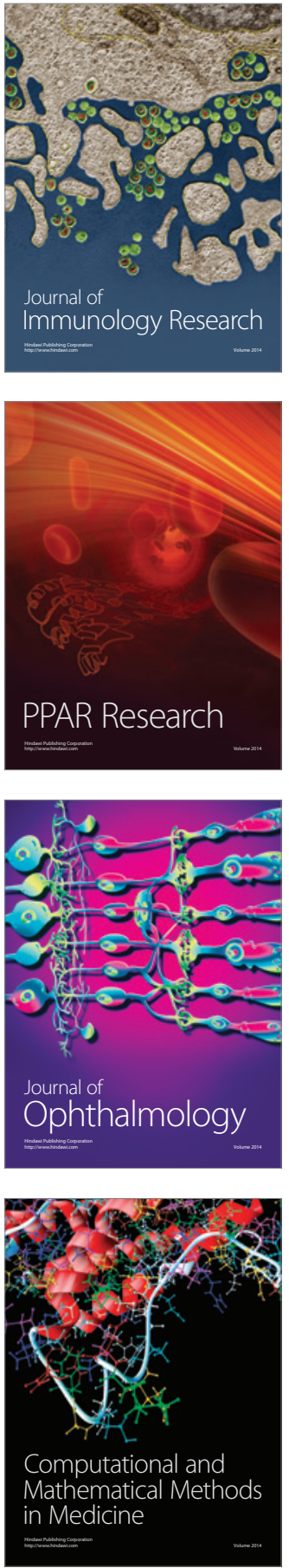

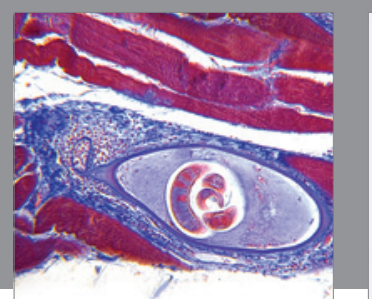

Gastroenterology Research and Practice

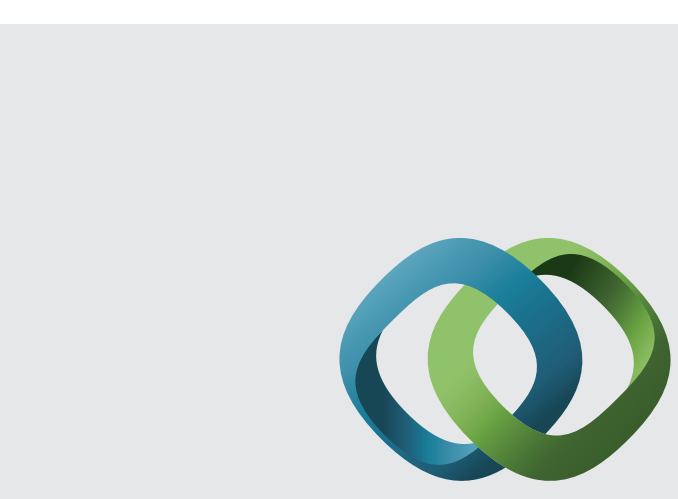

\section{Hindawi}

Submit your manuscripts at

http://www.hindawi.com
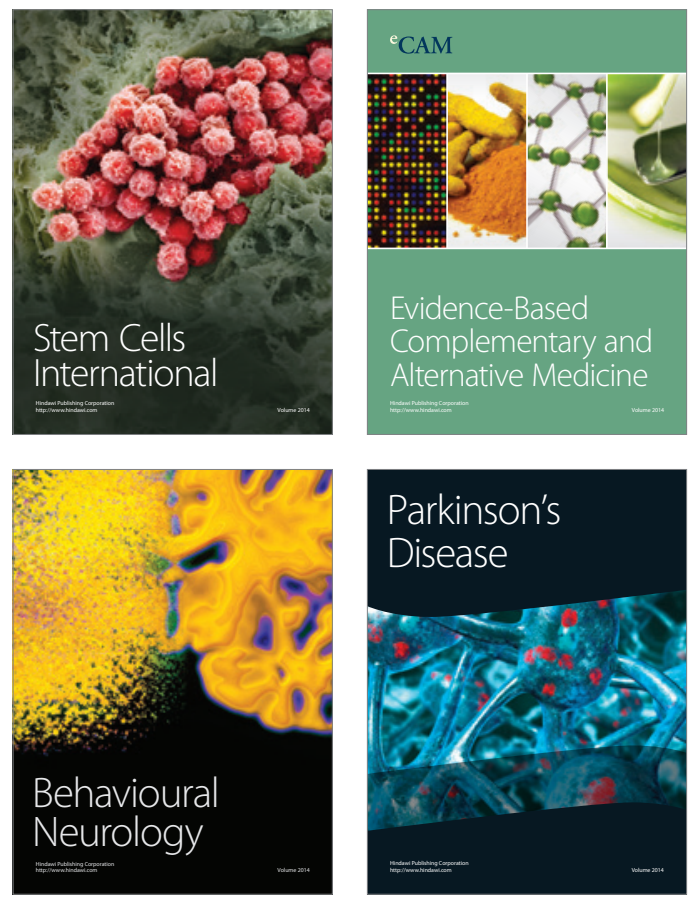
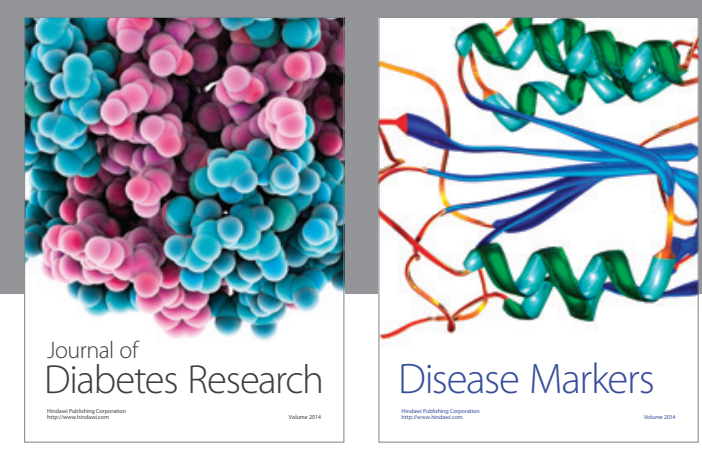

Disease Markers
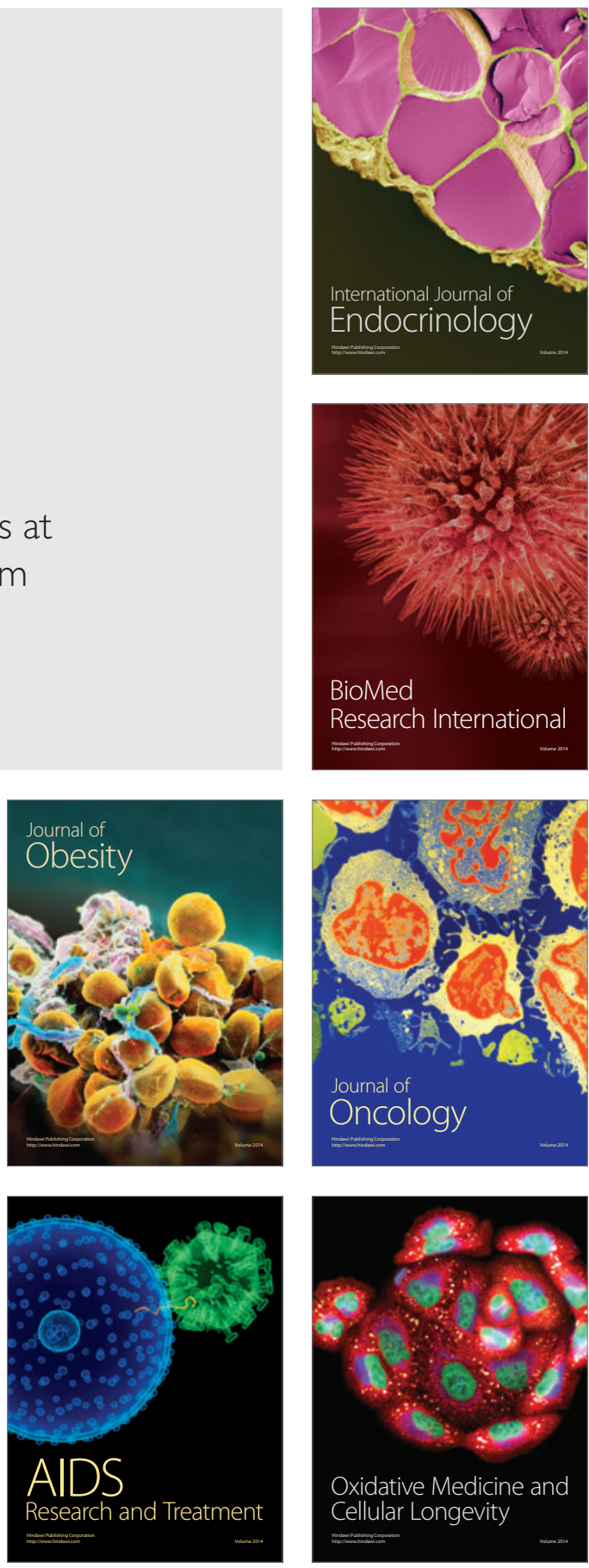